\title{
Koronaratherosklerose bei der Rheumatoiden Arthritis
}

\author{
Karpouzas GA et al. Impact of Cumulative \\ Inflammation, Cardiac Risk Factors, and \\ Medication Exposure on Coronary Atherosclero- \\ sis Progression in Rheumatoid Arthritis. Arthritis \\ Rheumatol 2020; 72: 400-408. doi: 10.1002/ \\ art. 41122
}

Patienten mit einer Rheumatoiden Arthritis (RA) haben im Vergleich zu Gesunden ein deutlich erhöhtes Risiko für kardiovaskuläre Komplikationen. Wie viele Betroffene entwickeln im Krankheitsverlauf Koronarplaques? Wie setzen sich diese atherosklerotischen Läsionen zusammen, wie verändern sie sich und welche Faktoren beeinflussen ihr Voranschreiten?

Diesen Fragen ging ein US-Forscherteam nach. Die Wissenschaftler werteten die Daten von 101 RA-Patienten aus, die zwischen 2010 und 2011 im Rahmen einer Studienteilnahme mittels CT-Koronarangiografie untersucht worden waren. Alle Patienten waren zum Untersuchungszeitpunkt beschwerdefrei und wiesen keine kardiovaskuläre Vorbelastung auf. Nach einem Zeitraum von durchschnittlich $83 \pm 3$,6 Monaten unterzogen die Forscher die Studienteilnehmer einer Folgeuntersuchung. Anhand der Bildgebung objektivierten sie die Veränderungen der Koronarplaques im Zeitverlauf. Hierbei wendeten sie einerseits den sogenannten Segment-InvolvementScore (Gesamtzahl der Plaque-belasteten Koronarsegmente) und andererseits den Segment-Stenose-Score (kumulativer Stenosegrad in allen beurteilbaren Segmenten). Ferner klassifizierten sie die Zusammensetzung der Atheroskleroseläsionen als „kalzifiziert“, „nicht kalzifiziert“ bzw. „gemischt" und quantifizierten den Kalzifizierungsgrad mithilfe des Agatston-Scores. Schließlich prüften die Forscher, welche Faktoren für ein Voranschreiten der Plaques prädisponieren. Die hierbei analysierten Parameter umfassten neben kardiovaskulären Risikofaktoren (Bluthochdruck, Diabetes mellitus, Hyperlipidämie, Rauchen) die Krankheitsaktivität sowie die Behandlung mit Kortikosteroiden, konventionellen bzw. biologischen DMARDs (disease modifying drugs) und Statinen. 


\section{Ergebnisse}

Das Durchschnittsalter des Studienkollektivs betrug 51 Jahre, $86 \%$ waren Frauen. 70 Patienten (69,3\%) wiesen bereits zum Zeitpunkt der Erstuntersuchung Koronarplaques in insgesamt 187 Segmenten auf. Anhand der Folgebildgebung diagnostizierten die Wissenschaftler bei $48 \%$ der Patienten einen Plaqueprogress. Dabei beobachteten sie in insgesamt 97 zuvor Plaque-freien Segmenten neue Atheroskleroseläsionen: 15 bei 10 initial Plaque-unbelasteten und 82 bei 37 -vorbelasteten Patienten. 20 der 97 neu aufgetretenen Plaques waren nicht kalzifiziert, 56 waren kalzifiziert und 21 waren gemischt. Patienten mit einem Voranschreiten der Koronarkalzifikationen waren älter, häufiger adipös, litten häufiger an einer Hypertonie und wiesen im Vergleich zu den Patienten ohne Progress eine höhere kumulative Inflammation (zeitlich gemittelte CRP-Level) auf. Den Berechnungen der Wissenschaftler zu Folge beugte eine längere Behandlung mit biologischen DMARDs dem Voranschreiten nicht kalzifizierter Plaques sowie einem Remodeling der Läsionen vor und schützte insbesondere Patienten ohne initiale Koronarkalzifikationen vor entsprechenden Veränderungen - und zwar unabhängig von der inflammatorischen Belastung, der Prednisondosis sowie der Statinexposition. Eine längere Statinbehandlung beugte ebenfalls dem Voranschreiten nicht kalzifizierter Plaques vor und schwächte die negativen Auswirkungen der Inflammation auf das Plaquewachstum und die Koronarkalzifikation ab. Auch eine strenge Kontrolle des systolischen Blutdrucks verringerte die negativen Folgen der Inflammation bezüglich des Plaquewachstums.

\section{FAZIT}

Ein erheblicher Anteil der RA-Patienten weist okkulte Koronarplaques auf, schlussfolgern die Autoren. Die Inflammation stellt dabei einen unabhängigen Prädiktor für ein Voranschreiten der Läsionen dar. Die dauerhafte Minimierung der Entzündungsbelastung muss daher, so ihr Fazit, auch unter kardiovaskulären Aspekten ein wesentliches Therapieziel sein. Ferner scheinen Biologika und Statine sowie eine strenge Blutdruckkontrolle dem Plaquewachstum entgegenzuwirken.

Dr. med. Judith Lorenz, Künzell 